Return to the Manage Active Submissions page at http://spie.org/submissions/tasks.aspx and approve or disapprove this submission. Your manuscript will not be published without this approval. Please contact author_help@spie.org with any questions or concerns.

\title{
Bending Loss Improvement and Twisting Loss Studies of Flexible Multimode Polymer Waveguides
}

\author{
F. Shi, N. Bamiedakis, R. V. Penty, I. H. White, D. Chu* \\ Electrical Engineering Division, Engineering Department, University of Cambridge, \\ 9 J. J. Thomson Avenue, Cambridge, CB3 OFA, UK \\ *dpc31@ cam.ac.uk
}

\begin{abstract}
Multimode polymer waveguides have attracted considerable interest for use in high-speed on-board communication links as they provide low loss $(<0.04 \mathrm{~dB} / \mathrm{cm}$ at $850 \mathrm{~nm})$ and high bandwidth $(>40 \mathrm{GHz} \times \mathrm{m})$ and can be cost-effectively integrated onto standard PCBs. The fabrication of such waveguides on flexible substrates can provide additional advantages: shape flexibility, lightweight and reduced thickness which are particularly important in the aviation and automotive industries. Such flexible and lightweight optical connections will play an important role in next-generation airplanes and driverless cars connecting the multitude of peripheral sensors with the central processing unit at high speed and low latency. However, in such applications, flexible polymer waveguides are required to be bent to meet their stringent space requirements and twisted or stretched when connecting movable parts. Under sharp flexure, the bending or twisting loss dominates the waveguide loss limiting their practical use. In this work therefore, we present a new waveguide design for flexible polymer waveguides with improved bending performance and derive useful layout rules for minimizing twisting losses in such samples. The proposed waveguide structure only requires one additional fabrication step and achieves bending losses below $0.5 \mathrm{~dB}$ for a $3 \mathrm{~mm}$ bend. In comparison, the conventional waveguide design yields a $2 \mathrm{~dB}$ loss under the same bending radius and launch condition. Additionally, useful equations relating the maximum allowed number of twisting turns for low excess loss with sample thickness and width are proposed. Bending and twisting measurements on flexible waveguide samples are presented validating these methods and demonstrating the potential of this technology.
\end{abstract}

Keywords: optical interconnects, polymer waveguides, flexible waveguides, waveguide bends, waveguide twisting.

\section{INTRODUCTION}

Optical interconnects have attracted great interest for use inside high-performance electronic systems, such as data centres, data storage systems and supercomputers, as they can provide significant performance advantages over conventional electrical interconnects: higher bandwidth, improved power efficiency, increased density and reduced thermal management requirements [1-3]. Various optical technologies have been developed for use at the different interconnection levels such as board-to-board, chip-to-chip and on-chip. Multimode polymer waveguides in particular, are an attractive technology for use in board-level interconnects (on-board links in the range 1 to $100 \mathrm{~cm}$ ) as it allows the direct integration of high-speed optical links on conventional printed circuit boards (PCBs) and enables the formation of low-cost large-capacity optical backplanes [4-5]. Considerable research work has been carried out in recent years on polymer waveguides [5-7] and numerous optical backplanes based on polymer multimode waveguides have been successfully demonstrated [8-10]. A leading class of such polymetric optical waveguides suitable for board-level integration are siloxane-based waveguides [11,12] as they have been proved to exhibit excellent mechanical and optical properties. More specifically, siloxane materials exhibit resistance to high temperatures up to $300^{\circ} \mathrm{C}$, environmental stability, long lifetimes and low material absorption $(\sim 0.04 \mathrm{~dB} / \mathrm{cm})$ at the datacommunications' wavelength of $850 \mathrm{~nm}$. In addition, they can be deposited on both flexible and rigid substrates and patterned with a variety of methods [11, 13]. Large-size waveguides with core dimensions ranging from 30 to $70 \mu \mathrm{m}$ are typically employed for the formation of onboard optical interconnects, as they provide relaxed alignment tolerance $(50-\mu \mathrm{m}$ wide waveguides offer $1 \mathrm{~dB}$ alignment tolerances of $\pm 10 \mu \mathrm{m}$ [4]) and enable cost-effective board assembly with standard pick-and-place tools [14]. The bandwidth performance of these polymer waveguides has also been studied and the results indicate that they exhibit 
Return to the Manage Active Submissions page at http://spie.org/submissions/tasks.aspx and approve or disapprove this submission. Your manuscript will not be published without this approval. Please contact author_help@spie.org with any questions or concerns.

relatively large bandwidth-length products in excess of $40 \mathrm{GHz} \times \mathrm{m}$ despite their highly multimode nature [8]. Data transmission at $40 \mathrm{~Gb} / \mathrm{s}$ using non-return-to-zero (NRZ) modulation over a $1 \mathrm{~m}$ long spiral multimode polymer waveguide fabricated both on a rigid [15] and flexible substrate [16] has been successfully demonstrated. $56 \mathrm{~Gb} / \mathrm{s}$ data transmission using 4-level pulse-amplitude modulation (PAM-4) has also been demonstrated over the $1 \mathrm{~m}$ long spiral waveguide [17].

The further formation of siloxane-based polymer waveguides on flexible substrates provides significant benefits over the conventional rigid structures. Flexible polymer waveguides form thin, lightweight and bendable optical interconnects which can be deployed in applications where shape and weight are important such as in next-generation cars and airplanes. However, excess loss due to bending or twisting can severely impact the performance of such bendable waveguides and limit their practical use. As a result, a few studies on the performance of such waveguides under flexure have been recently reported [6, 18-20]. For example, in [18], bending loss measurements on flexible multimode waveguides using a $62.5 \mu \mathrm{m}$ multimode fibre (MMF) input indicated that no significant bending loss is induced over a $360^{\circ}$ bend for bending radii larger than $30 \mathrm{~mm}$. In [19], bending loss studies were carried out on flexible waveguides, demonstrating a $4.3 \mathrm{~dB}$ excess loss for $9 \mu \mathrm{m}$ SMF input when the sample is bent with a $5 \mathrm{~mm}$ radius. Similar studies have been reported in [20]. In [6] we demonstrated that bending losses below $2 \mathrm{~dB}$ can be achieved with a $50 \mu \mathrm{m}$ MMF input for a $3 \mathrm{~mm}$ radius for siloxane-based flexible waveguides. Additionally, it was shown that very low excess loss of $0.1 \mathrm{~dB}$ is obtained due to twisting for a SMF and $50 \mu \mathrm{m}$ MMF inputs for up to 4 full twists turns as long as the lateral tension was minimised.

In this paper, we propose a new design for flexible polymer waveguides which can significantly improve their bending loss performance and provide useful rules for estimating allowed twisting of such samples. The proposed new design allows a low excess bending loss of $0.5 \mathrm{~dB}$ for a $3 \mathrm{~mm}$ bend under $50 \mu \mathrm{m}$ MMF. In comparison, the conventional design yields a $2 \mathrm{~dB}$ loss under the same launch condition. As for the twisting loss performance, useful design rules are provided which can be used to calculate the maximum allowed number of twisting turns as a function of the sample's geometric parameters and applied force. Relevant experiments are reported which validate these.

\section{MULTIMODE POLYMER WAVEGUIDE TECHNOLOGY}

The flexible polymer waveguides used in this work are fabricated from siloxane materials developed by Dow Corning: Dow Corning ${ }^{\circledR}$ WG-1020 Optical Elastomer (core) and XX-1023 Optical Elastomer (cladding). These materials exhibit the essential mechanical and optical properties for direct integration onto PCBs and flexible substrates such as low Young's modulus, low loss at the datacommunications' wavelength of $850 \mathrm{~nm}(<0.04 \mathrm{~dB} / \mathrm{cm})$, resistivity to hightemperature environments $\left(>300^{\circ} \mathrm{C}\right)$ required for PCB solder reflow and lamination, and long-term stability [21, 22]. These siloxane materials can be deposited by various methods (doctor-blading, spin coating, etc.) on both rigid and flexible substrates such as FR4, silicon, glass and polyimide. The refractive index of the core and cladding materials is respectively 1.5255 and 1.5104 at the $850 \mathrm{~nm}$ wavelength. The refractive index (RI) profile of the core can also be engineered into a step-index (SI) or graded-index (GI) profile by controlling the fabrication parameters [23]. In this work, the waveguide is designed to have a cross section of $\sim 50 \times 50 \mu \mathrm{m}^{2}$ which matches the core diameter of standard 50 $\mu \mathrm{m}$ MMF and a pitch value of $250 \mu \mathrm{m}$ which matches the pitch of vertical-cavity surface-emitting laser (VCSEL) arrays and MMF ribbons. The flexible polymer waveguide samples used in this work are fabricated on $125 \mu \mathrm{m}$-thick polyimide substrates using conventional photolithography. Two flexible samples each containing an array of 12 parallel waveguides but with different dimensions (sample A: $13.5 \times 10 \mathrm{~cm}^{2}$ and B: $7 \times 10 \mathrm{~cm}^{2}$ ) are fabricated under similar conditions (Figure 1). The sample features two slightly different waveguide designs which are presented in the following section.

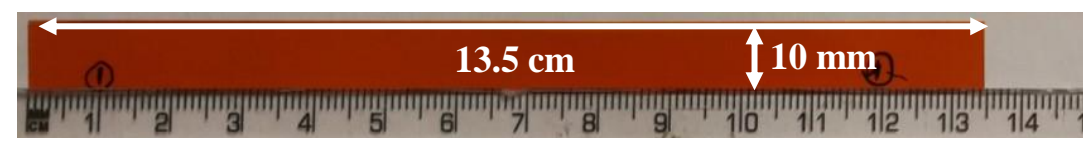

Figure 1. Image of flexible polymer waveguide samples with dimensions $135 \times 10 \mathrm{~mm}^{2}$. 
Return to the Manage Active Submissions page at http://spie.org/submissions/tasks.aspx and approve or disapprove this submission. Your manuscript will not be published without this approval. Please contact author_help@spie.org with any questions or concerns.

\section{BEND-INSENSITIVE FLEXIBLE POLYMER WAVEGUIDES}

Bending loss is one of the most important performance factors for flexible polymer waveguides. Currently, the best bending loss performance that could be achieved with this siloxane waveguide technology and the conventional waveguide design is $2 \mathrm{~dB}$ excess loss for a standard $50 \mu \mathrm{m}$ MMF input at a $2 \mathrm{~mm}$ radius [6]. Here, we propose a new waveguide design that offers improved bending loss performance from flexible polymer waveguide samples. Figure 2 shows the conventional and newly proposed waveguide design. There are two differences between the proposed bendinsensitive waveguides and the conventional design. Firstly, an additional layer is introduced in the bottom cladding with a RI lower than that of the siloxane cladding material in close proximity to the waveguide cores. Secondly, the thickness of the top cladding is significantly reduced to allow better light confinement in the waveguide core. The presence of the thin siloxane bottom and top cladding is the new design is essential to ensure that the light transmission properties of the waveguides remain un-affected if no bending is applied. These two features can be easily achieved in practice by introducing two additional steps in the fabrication process of conventional flexible waveguides. Here, PDMS is used as the material for lower RI material. Its RI at $850 \mathrm{~nm}$ is 1.41 .

(a)

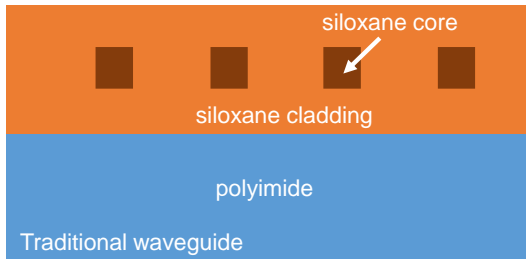

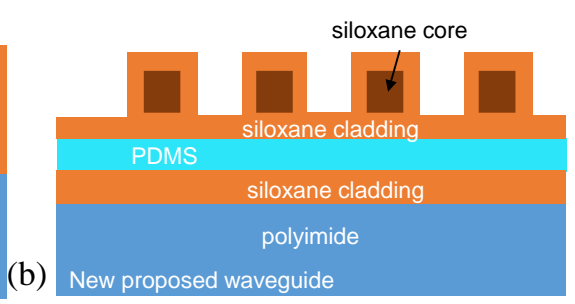

(b)

Figure 2. Waveguide with (a) the conventional and (b) the new proposed design.

Figure 3 shows the top and cross-section view of the fabricated flexible waveguide samples featuring the new bendinsensitive design. The waveguide facets are exposed with a dicing saw. The waveguide height is measured to be $\sim 52$ $\mu \mathrm{m}$ while the thickness of the bottom PDMS layer is $\sim 20 \mu \mathrm{m}$. It is hard to observe the bottom siloxane cladding layer as it is very thin $(\sim 5 \mu \mathrm{m})$. Siloxane cladding tails can be observed around the waveguide core due to the coating of the thin top cladding layer. Due to gravity, the cladding material tends to accumulate at the bottom of the vertical waveguide sidewalls before exposure, resulting in the tails observed in Figure 3. There is no simple and efficient method to remove these, but they should not affect the bending performance of the waveguides as the bending is introduced in the vertical direction (out-of-plane with respect to the waveguide array).

Top view

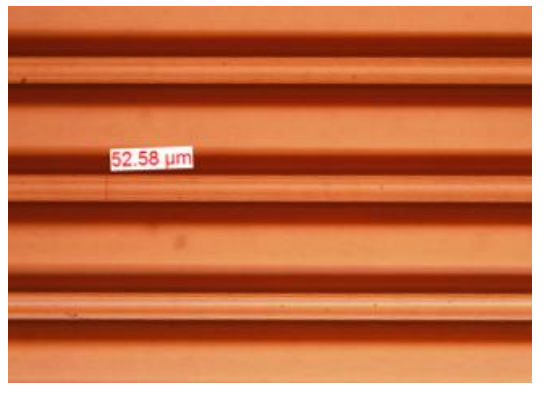

Cross-section view

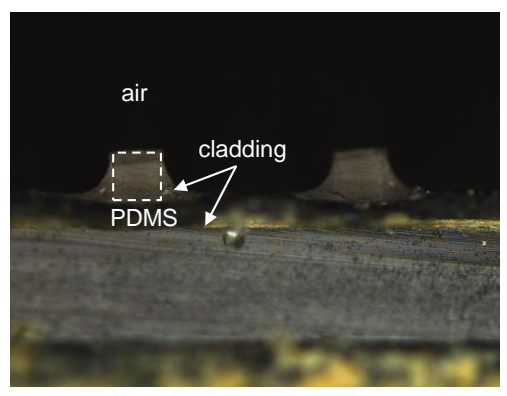

Figure 3. Top view and cross-section view of the fabricated bend-insensitive waveguide sample.

\subsection{Employed launch condition}

The bending performance of multimode polymer waveguides depends strongly on the employed launch condition as different waveguide modes exhibit different loss along the waveguide bend. In this work, a standard 50/125 $\mu \mathrm{m}$ MMF 


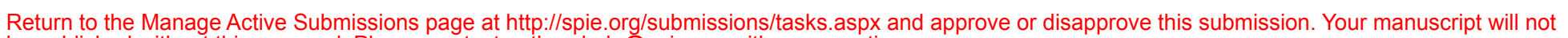
be published without this approval. Please contact author_help@spie.org with any questions or concerns.

input is employed as it is a widely used launch and its numerical aperture (NA) matches that of the waveguides. Figure 4 shows the normalised far-field beam profile at the output of the fibre and near-field image. The 5\% far-field angle is used to estimate the fibre NA. It is found to be $11.5^{\circ}$, which matches the NA of the fabricated waveguides $\left(12.3^{\circ}\right)$.

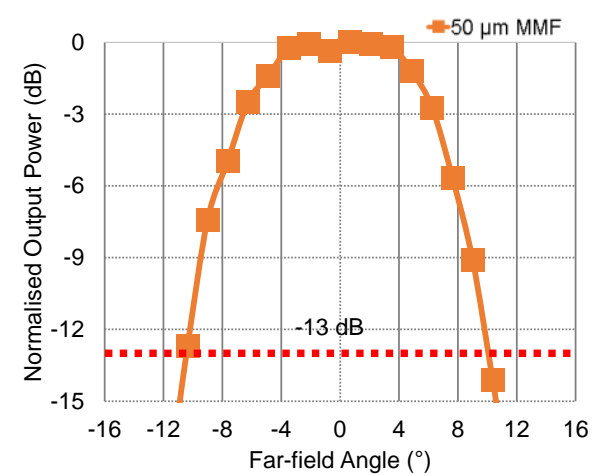

(a)

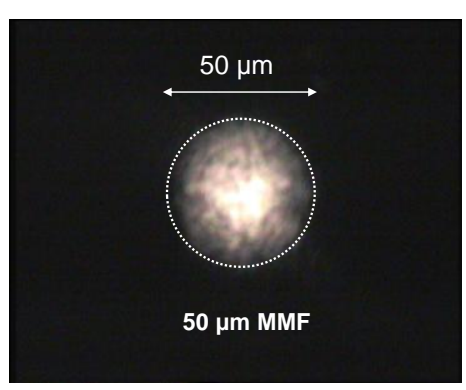

(b)

Figure 4. (a) Normalised far-field intensity profile and (b) near-field image of the 50/125 $\mu \mathrm{m}$ MM input used in the bending loss measurements. The red dashed line indicates the $-13 \mathrm{~dB}$ level which corresponds to the $5 \%$ far-field intensity.

\subsection{Bending loss performance}

Bending loss measurements are carried out on the flexible waveguides featuring the new design and are compared with these obtained from samples with the conventional one. The experimental setup is shown in Figure 5(a). An $850 \mathrm{~nm}$ VCSEL is employed as the light source and the emitted light is coupled into a $50 \mu \mathrm{m}$ MMF patchcord via a pair of $10 \times$ microscope objectives. A second $50 \mu \mathrm{m}$ MMF patchcord with a cleaved end is used to couple the light into the waveguides on the flexible sample. The waveguide sample is bent $180^{\circ}$ around a cylindrical mandrel of constant radius. At the output side, a $16 \times$ microscope objective is used to collect the transmitted light and focus it onto an optical detector. The numerical aperture (NA) of the employed lens at the waveguide output is appropriately chosen to be larger than that of the waveguide which ensures the collection of all transmitted light. At the input side, index matching gel is applied at the waveguide input to minimise Fresnel loss and scattering due to facet surface roughness. A near-field image of the output of the waveguide with the new design is shown in Figure 5(b). For each sample, 6 waveguides are tested and the average insertion loss is obtained for each bending radius.
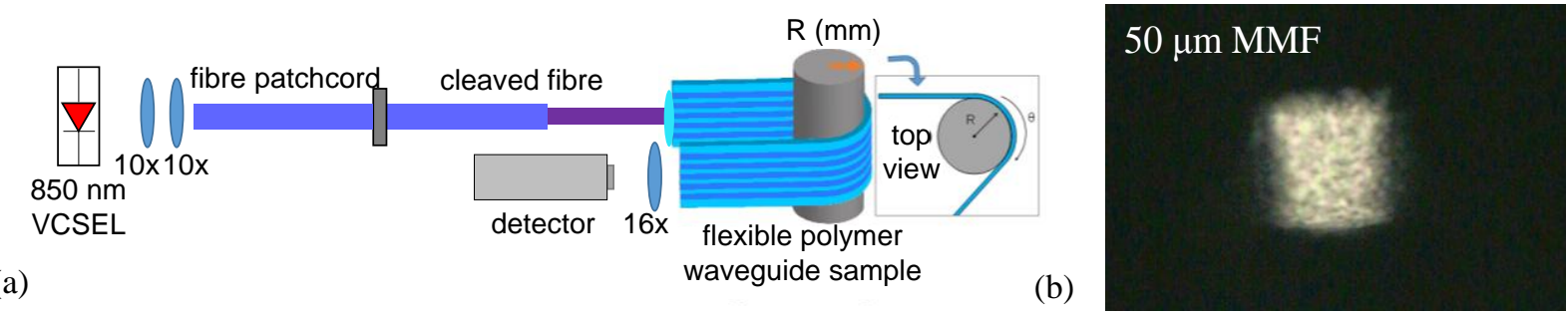

Figure 5 (a) Experimental system setup for bending loss studies and (b) near-field image of the bend-insensitive waveguide facet under $50 \mu \mathrm{m}$ MMF input launch.

Due to the asymmetry of the waveguide structure, out-of-plane sample bending along the vertical axis results in a different bending loss performance depending on the bending direction: top (towards the air) or bottom (towards the substrate). As a result, both cases are measured and the excess bending loss is compared with that obtained for a waveguide sample with the conventional design. The results obtained are shown in Figure 6 . The results show that excess bending loss for the waveguides with the conventional design is much higher than that obtained with the new design. In addition, bending towards the substrate results in lower excess losses as the air acts as the cladding layer, which has a larger refractive index difference with the core. The excess bending loss for a $3 \mathrm{~mm}$ bend is $2 \mathrm{~dB}$ for the 
Return to the Manage Active Submissions page at http://spie.org/submissions/tasks.aspx and approve or disapprove this submission. Your manuscript will not be published without this approval. Please contact author_help@spie.org with any questions or concerns.

traditional design, while for the new design it is found to be 1 and $0.5 \mathrm{~dB}$ for upward (towards the air) and downward (towards the substrate) bending respectively. A great improvement of the bending loss performance is therefore achieved. The excess bending loss for the new design and downward bending is found to be less than $1 \mathrm{~dB}$ even for a 2 mm radius.

(a)
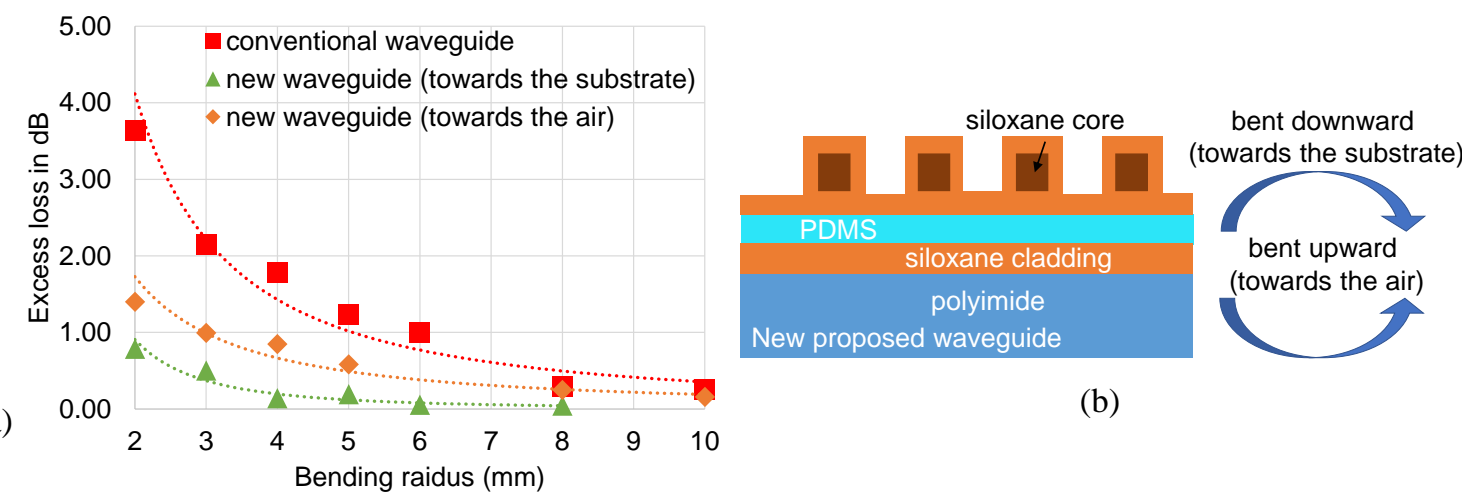

(b)

Figure 6. (a) Excess bending loss of the waveguides on flexible samples for a $50 \mu \mathrm{m}$ MMF input and (b) schematic illustration of the bending directions.

\section{TWISTING LOSS PERFORMANCE INVESTIGATION}

Twisting loss performance is another important characteristic of flexible waveguide samples. The sample dimensions (thickness, length, width), applied force, and number of twisting turns affect the shape of the sample that is generated due to the twisting and therefore the induced twisting loss [24]. Loss measurements are carried out on a flexible polymer waveguide sample with dimensions of $10 \mathrm{~cm}$ in length, $1 \mathrm{~cm}$ in width and $0.02 \mathrm{~cm}$ in thickness under the same $50 \mu \mathrm{m}$ MMF launch. Both sample ends are fixed on mounts and positioned on translational stages. One end is rotated perpendicular to the sample longitudinal axis a given number of twisting turns and pulled away as much as possible (without damaging the sample) in order to ensure that the twisted sample is fully stretched. 1 full twisting turn corresponds to $360^{\circ}$ rotation. Figure 7 shows sample images of the generated sample shape for different number of twisting turns. Due to the lateral tension on the flexible sample and its width, a twisting node (self-contact) is generated when two or more twisting turns are applied. This twisting node significantly changes the optical transmission path and introduces large twisting loss
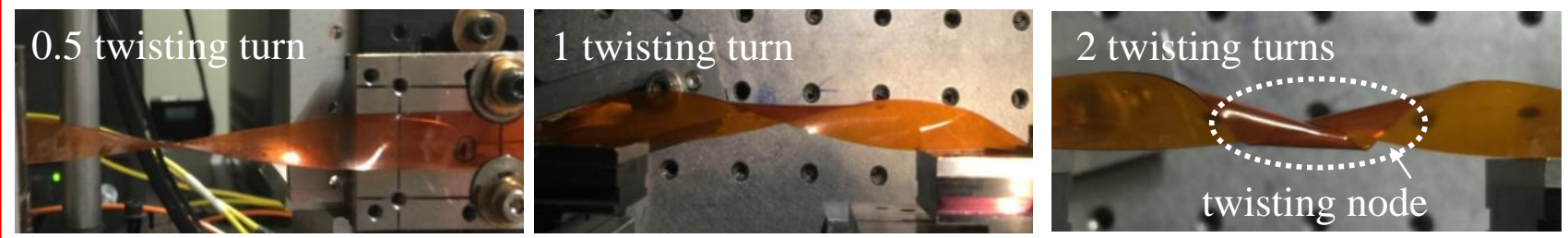

Figure 7. twisting patterns of the flexible polymer waveguide sample for different numbers of full twisting turns.

The measured excess twisting loss versus the number of twisting turns is given in Figure 8. It can be clearly noticed that the loss behaviour of the twisted sample can be divided into 2 parts. Twisting the sample up to 1 full twisting turn results in very low excess loss of $\sim 0.1 \mathrm{~dB}$, whereas, for over 1 full twisting turns, the loss jumps up to $\sim 1.7 \mathrm{~dB}$ due to the generation of the twisting node. The twisting node essentially results in tight waveguide bending over the node area, which generates the large additional loss. Once a twisting node has been generated, the excess twisting loss remains relatively stable regardless of the number of twisting turns (for the same applied force) as the tight bending does not change. 
Return to the Manage Active Submissions page at http://spie.org/submissions/tasks.aspx and approve or disapprove this submission. Your manuscript will not be published without this approval. Please contact author_help@spie.org with any questions or concerns.

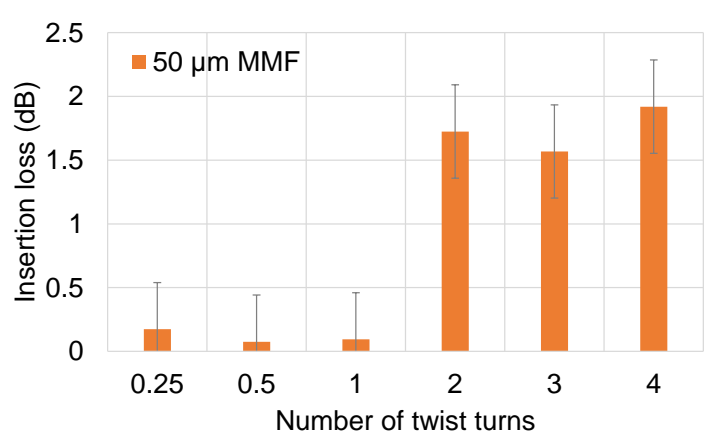

Figure 8. Excess twisting loss for the $10 \mathrm{~cm}$-long flexible polymer waveguide sample as a function of the number of twisting turns.

Detailed studies on the shape generated in flexible ribbons due to twisting can be found in [24, 25]. These can be categorised in helicoids, wrinkles and loops (nodes) and depend on the sample geometric parameters (length, width, thickness), applied lateral force, and number of twisting turns. Using the equations provided in [24], phase diagrams can be plotted that determine the generated shape of the flexible sample due to twisting. The phase diagram for the flexible waveguide sample used in the twisting measurements is calculated and shown in Figure 9. The x-axis represents the lateral force applied while the y-axis the number of full twisting $\left(360^{\circ}\right)$ turns. Given that the additional loss induced due to twisting is very small if the twisting node is not present, for the twisting loss studies carried out here, we are only interested in the area of the phase diagram that indicates the generation of the twisting node (self-contact). This area is defined with the red line in Figure 9. Above the red line, the twisted waveguide sample will be in self-contact generating a twisting node and whereas below the red line a helicoid shape will be produced, and the sample will be node-free.

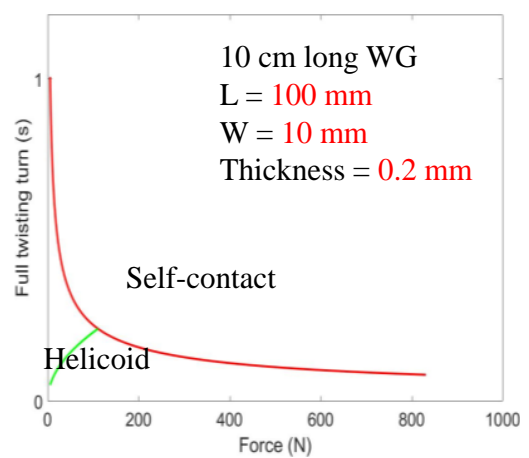

Figure 9. Phase diagram of the flexible polymer waveguide samples under twist.

It can be clearly seen from the diagram that for the flexible sample used in the experiments, self-contact (i.e. a twisting node is formed) is expected if the sample is twisted more than 1 full twisting turn irrespective of the lateral force applied. This agrees with the experimental observations. These phase diagrams therefore can allow the estimation of the maximum allowed twisting turns for a particular flexible waveguide sample.

\section{CONCLUSIONS}

The bending and twisting loss performance of flexible polymer waveguides is an important parameter for their realworld deployment. In this paper, we investigate the bending loss performance of flexible waveguide samples and propose a new waveguide design which can significantly improve it. It is experimentally shown that this new design allows a low excess bending loss of $0.5 \mathrm{~dB}$ for a $3 \mathrm{~mm}$ radius under a $50 \mu \mathrm{m}$ MMF launch whilst the conventional waveguide yields a $2 \mathrm{~dB}$ loss for the same radius and launch condition. In addition, we derive useful layout rules that relate twisting losses with the sample geometric parameter and prove that twisting results only in very low excess loss provided that the formation of twisting node is avoided. 
Return to the Manage Active Submissions page at http://spie.org/submissions/tasks.aspx and approve or disapprove this submission. Your manuscript will not be published without this approval. Please contact author_help@spie.org with any questions or concerns.

\section{ACKNOWLEDGEMENTS}

The authors would like to acknowledge Dow Corning for the provision of the polymer waveguide samples and Jaguar Land Rover for supporting this work via the CAPE LEASE project.

\section{REFERENCES}

[1]. D. Miller, "Device Requirements for Optical Interconnects to Silicon Chips," Proceedings of the IEEE, vol. 97, pp. 1166-1185, 2009.

[2]. C. Hoyeol, et al. "Power comparison between high-speed electrical and optical interconnects for interchip communication," Journal of Lightwave Technology, vol. 22, pp. 2021-2033, 2004.

[3]. M. A. Taubenblatt, "Optical Interconnects for High-Performance Computing," Journal of Lightwave Technology, vol. 30, pp. 448-457, 2012.

[4]. N. Bamiedakis, et al. "Low-Cost PCB-Integrated 10-Gb/s Optical Transceiver Built With a Novel Integration Method," IEEE Transactions on Components, Packaging and Manufacturing Technology, vol. 3, pp. 592-600, 2013.

[5]. R. Dangel, et al., "Polymer waveguides for electro-optical integration in data centers and high-performance computers," Optics Express, vol. 23, pp. 4736-4750, 2015.

[6]. F. Shi, et al., "Flexible Multimode Polymer Waveguide Arrays for Versatile High-Speed Short-Reach Communication Links," Journal of Lightwave Technology, vol. 36, pp. 2685-2693,2018.

[7]. R. Dangel, et al., "Development of Versatile Polymer Waveguide Flex Technology for Use in Optical Interconnects," Journal of Lightwave Technology, vol. 31, pp. 3915-3926, 2013.

[8]. N. Bamiedakis, et al. "A $40 \mathrm{~Gb} / \mathrm{s}$ Optical Bus for Optical Backplane Interconnections," Journal of Lightwave Technology, vol. 32, pp. 1526-1537,2014.

[9]. K. Schmidtke, et al., "960 Gb/s Optical Backplane Ecosystem Using Embedded Polymer Waveguides and Demonstration in a 12G SAS Storage Array," Journal of Lightwave Technology, vol.31, pp. 3970-3975, 2013.

[10]. J. Beals, et al. "A terabit capacity passive polymer optical backplane based on a novel meshed waveguide architecture," Appl. Phys. A: Mater. Sci. Process., vol. 95, pp. 983-988, 2009.

[11]. J. V. DeGroot Jr., "Cost-effective optical waveguide components for printed circuit applications," in Passive Components and Fiber-based Devices IV (SPIE), pp. 1-12, 2007.

[12]. R. S. E. John, et al., "Thermally Stable, Low Loss Optical Silicones: A Key Enabler for Electro-Optical Printed Circuit Boards," Journal of Lightwave Technology, vol. 33, pp. 814-819, 2015.

[13]. B. W. Swatowski, et al., "Flexible, stable, and easily processable optical silicones for low loss polymer waveguides," Proc. SPIE 8622, Organic Photonic Materials and Devices XV, pp. 1-11, 2013.

[14]. N. Bamiedakis, et al., "Cost-Effective Multimode Polymer Waveguides for High-Speed On-Board Optical Interconnects," IEEE Journal of Quantum Electronics, vol. 45, pp. 415-424, 2009.

[15]. N. Bamiedakis, et al., "40 Gb/s Data Transmission Over a 1-m-Long Multimode Polymer Spiral Waveguide for Board-Level Optical Interconnects," Journal of Lightwave Technology, vol. 33, pp. 882-888, 2015.

[16]. N. Bamiedakis, et al., "High-Speed Data Transmission Over Flexible Multimode Polymer Waveguides Under Flexure." IEEE Photonics Technology Letters, vol. 30, pp. 1329-1332, 2018.

[17]. N. Bamiedakis, et al., "56 Gb/s PAM-4 Data Transmission Over a 1 m Long Multimode Polymer Interconnect," in Conference on Lasers and Electro-Optics (CLEO)(Optical Society of America, San Jose, California, 2015), p. STu4F.5.

[18]. R. Dangel, et al. "Development of Versatile Polymer Waveguide Flex Technology for Use in Optical Interconnects," Journal of Lightwave Technology, vol. 31, pp. 3915-3926, 2013.

[19]. X. Dou, et al. "Highly flexible polymeric optical waveguide for out-of-plane optical interconnects," Optics Express, vol. 18, pp. 16227-16233, 2010.

[20]. R. Bruck, et al. "Flexible thin-film polymer waveguides fabricated in an industrial roll-to-roll process," Applied Optics, vol. 52, pp. 4510-4, 2013.

[21]. R. S. E. John, et al., "Thermally Stable, Low Loss Optical Silicones: A Key Enabler for Electro-Optical Printed Circuit Boards," Journal of Lightwave Technology, vol. 33, pp. 814-819, 2015.

[22]. B. W. Swatowski, et al., "Flexible, stable, and easily processable optical silicones for low loss polymer waveguides," Proc. SPIE 8622, Organic Photonic Materials and Devices XV, pp. 1-11, 2013.

[23]. B. W. Swatowski, et al. "Graded Index Silicone Waveguides for High Performance Computing," in IEEE Optical Interconnects Conference (OIC), pp. 1-3, 2014.

11286 - 18 V. 1 (p.7 of 8) / Color: No / Format: A4 / Date: 1/14/2020 7:23:36 AM

SPIE USE: DB Check, Prod Check, Notes: 
Please verify that (1) all pages are present, (2) all figures are correct, (3) all fonts and special characters are correct, and (4) all text and figures fit within the red margin lines shown on this review document. Complete formatting information is available at http://SPIE.org/manuscripts

Return to the Manage Active Submissions page at http://spie.org/submissions/tasks.aspx and approve or disapprove this submission. Your manuscript will not be published without this approval. Please contact author_help@spie.org with any questions or concerns.

[24]. J. Chopin, et al. "Helicoids, Wrinkles, and Loops in Twisted Ribbons," Physical Review Letters, vol. 111, p. $174302,2013$.

[25]. J. Chopin, et al. "Roadmap to the Morphological Instabilities of a Stretched Twisted Ribbon," Journal of Elasticity, vol. 119, pp. 137-189, 2014. 